\title{
Utilizing Fundamental Beam-Mode Shaping Technique for Top-Hat Laser Intensities in Direct Laser Interference Patterning
}

\author{
Mikhael El-Khoury ${ }^{1}$, Bogdan Voisiat ${ }^{2}$, Tim Kunze ${ }^{1}$, and Andrés Fabián Lasagni ${ }^{1,2}$ \\ ${ }^{1}$ Fraunhofer Institute für Werkstoff und Strahltechnik IWS, Winterbergstrasse 28, 01277 Dresden, \\ Germany \\ E-mail: mikhael.el-khoury@iws.fraunhofer.de \\ ${ }^{2}$ Institut für Fertigungstechnik, Technische Universitaet Dresden, Zeunerbau, George-Bähr-Str. 3c, \\ 01069 Dresden, German
}

In this work, a diffractive fundamental beam-mode shaper (FBS) element is combined with a Direct Laser Interference Patterning (DLIP) optical setup to generate a square-shaped top-hat intensity distribution in the interference volume. The interference patterns produced by a symmetrical 4beam DLIP setup with Gaussian and Flat-top intensity distributions are measured and characterized. The impact of misalignments in the optical setup on the resulting intensity profile is analyzed and supplemented by theoretical calculations of the distorted interference patterns. Finally, top-hat shaped interference patterns can be introduced and utilized in order to generate surface patterns with improved homogeneity which eventually will lead to an improved performance in applications with functionalized surfaces.

DOI: $10.2961 /$ jlmn.2018.03.0021

Keywords: direct laser interference patterning, diffractive optics, fundamental beam-mode shaper, top-hat, aberrations, interference optics

\section{Introduction}

Laser surface texturing (LST) technologies are capable to improve different surface properties such as wettability, self-cleaning, tribological and antifouling [1-5]. This can be traced back to the capability of LST technologies to produce surfaces with well-controllable topographic characteristics. Out of the available LST methods, Direct Laser Interference Patterning (DLIP) has emerged as a promising technology capable to produce high resolution microtextures with high throughput $[6,7]$.

DLIP enables the direct fabrication of flexible periodic surface patterns with a well-defined long-range order based on interference principles. Laser material interaction occurs predominantly at the positions corresponding to the interference maxima, inducing various metallurgical processes such as melting, ablation and recrystallization [7, 9]. Since the local intensity at the interference maxima positions is directly connected to the intensity distribution in the laser beam profile, non-uniform surface textures may arise due to non-uniformities of the input laser beam.

Most commercial lasers provide beams with Gaussian or TEM00 intensity distribution. This intensity profile preserves its distribution during propagation and it can be focused to a diffraction-limited spot. However, the energy distribution of the Gaussian beam gradually decreases from the center to the periphery of the laser spot, so that only $36.8 \%$ of the pulse energy is used efficiently for the optimal focus spot size [10]. Consequently, nearly two-thirds of the used laser power located at the edges of the spot by simultaneously being above the threshold fluence level is wasted. This leads to unwanted damages of the surrounding material by uncontrolled melting and an enlarged heat- affected zone (HAZ) during DLIP. Another disadvantage of the Gaussian beam profile is the footprint of the ablated zone, which possesses a round shape and causes an inhomogeneous profile along the processing line [10]. This is usually circumvented by an increase of the pulse-to-pulse overlap which leads to an improved pattern homogeneity but at the same time to a low throughput $[6,11]$. Therefore, to decrease the HAZ and to improve throughput, reshaping of the Gaussian laser beam into a uniform square-shaped intensity distribution with sharp edges is required for DLIP processing which improves the throughput due to a substantially reduced pulse-to-pulse overlap.

This article describes the possibility to generate a square-beam with top-hat laser intensity distribution in the interference zone by using a fundamental beam-mode shaper (FBS) applied to a 4-beam DLIP setup. The adjustments of the system and the resulted interference patterns are examined. The origin of the appeared non-uniformity intensity distribution is determined experimentally and supplemented by simulation.

\section{Theoretical background \\ 2.1 Interference theory}

DLIP implements physical phenomenon of interference of coherent light waves to produce periodic structures on surface by transferring the pattern shape directly to the material [12-18]. The interference patterns are formed by splitting a coherent laser beam into multiple beams and hereafter overlapping them on the surface of the sample. The shape of the produced interference pattern is determined by the number of laser beams, their polarization, phase, energy and distribution [17-20]. There are two important require- 
ments for formation of periodic structures using the DLIP method. First, the material should absorb the energy at the selected laser wavelength and second, the laser should provide sufficient pulse energy for direct ablation or modification of the processed material.

Under the assumption of plane waves, the total electric field $(E)$ of the interference pattern can be obtained by the superposition of $N$ laser beams:

$$
\vec{E}(r)=\sum_{m=1}^{N} \vec{E}_{m}=\sum_{m=1}^{N} A_{m} \vec{p}_{m} \cos \left(k \vec{n}_{m} \vec{r} \pm 2 \pi v t+\delta_{m}\right),
$$

where $A_{m}$ is the amplitude, $\vec{p}_{m}$ is the polarization vector, $k=2 \pi / \lambda$ is the wave number, $\lambda$ is the wavelength, $v$ is the frequency and $\delta_{m}$ is the phase of the electric field, $\vec{n}_{m}$ is the unit vector in the propagation direction, $\vec{r}$ is the position vector [18].

Then, the total intensity of the pattern formed by interference of multiple waves with plane wave-fronts can be described as [21]:

$$
I=\frac{n c \varepsilon_{0}}{2}|E|^{2}
$$

where $n$ is refractive index, $c$ is the speed of light in vacuum and $\varepsilon_{0}$ is the permittivity of free space. For a 4-beam DLIP setup $(N=4)$, where each of the sub-beams is symmetrically distributed around the optical axis (see Fig. 1a), a periodic square-lattice shaped intensity distribution result (Fig. 1b). The period of the profile depends on the polar angle $\theta$ and the wavelength $\lambda$ according to:

$$
\Lambda_{4}=\frac{1}{\sqrt{2}} \frac{\lambda}{\sin (\theta)} \text {. }
$$
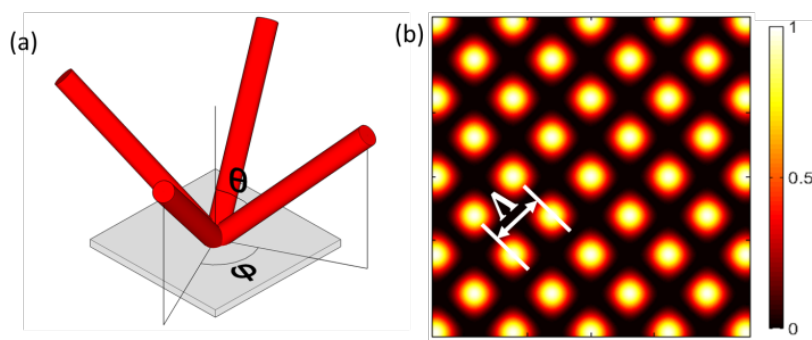

Fig. 1. (a) Sketch of four symmetrically distributed interfering sub-beams and (b) the resulting interference pattern distribution. The polar angle $\theta$ is the same for all four beams. The azimuthal angle $\phi$ between each consecutive beam is equal to $90^{\circ}$.

\subsection{Principle of fundamental beam-mode shaper}

The fundamental beam-mode shaper is a diffractive optical element which generates a square top-hat intensity distribution in the focal plane of the focusing lens by redistributing irradiance and phase of the wave front [22-25]. The depth of focus is maintained and comparable to the Rayleigh length of the focused Gaussian beam without the FBS element (see Fig. 2) [22, 24]. The efficiency of the FBS element is $95 \%$ in an area limited by $1 / \mathrm{e}^{2}$ level, where only $5 \%$ of the laser energy is wasted in the tail region. The areal uniformity of the top-hat profile is $\pm 2.5 \%$. The FBS approach requires specific input laser beam parameters. The input laser beam must of high beam quality $\left(T E M_{00}\right.$, $M^{2}<1.4$ ) with a particular beam diameter of $4 \mathrm{~mm}$ with $\pm 5 \%$ tolerance $[22,24]$.
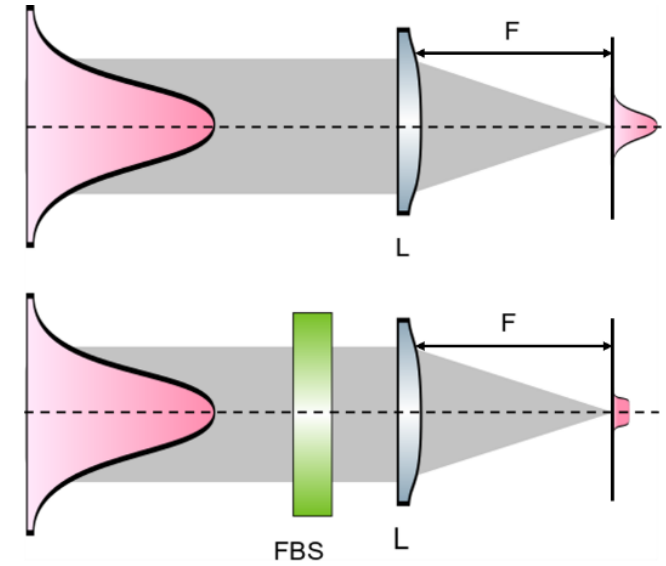

Fig. 2. Focused beam profile with and without FBS element [22].

\section{Experimental details}

A schematic representation of the experimental DLIP setup is shown in Fig. 3 which includes a nanosecond (ns)pulsed laser system (Laser Tech 1053 Advanced, Laser Export) working at $1053 \mathrm{~nm}$ with a maximum average output power of $4 \mathrm{~W}$ ( $4 \mathrm{kHz}$ repetition rate). The FBS beam shaper (TOPAG Lasertechnik GmbH) was integrated infront of the 4-beams DLIP optical configuration [26], which consists of a 4-beams splitter DOE (separation angle $13.58^{\circ} \times 13.58^{\circ}$ ), a pyramid prism (apex angle $138.4^{\circ}$ ) and a focussing lens $(\mathrm{EFL}=100 \mathrm{~mm})$. A confocal telescope with $(\times 4)$ magnification was used to provide a $4.0 \mathrm{~mm}$ beam diameter at the FBS element. Finally, a CCD profiling camera (Spiricon Laser Beam Diagnostics, SP620U) with beam profile analyzing software (Spiricon's BeamGage $\left.{ }^{\circledR}\right)$ was employed in order to characterize the resulting DLIP pattern intensity distributions. Additionally, a optical filter (OF) was utilized to reduce laser power before CCD profiling measurements (to prevent damages of the CCD sensor).

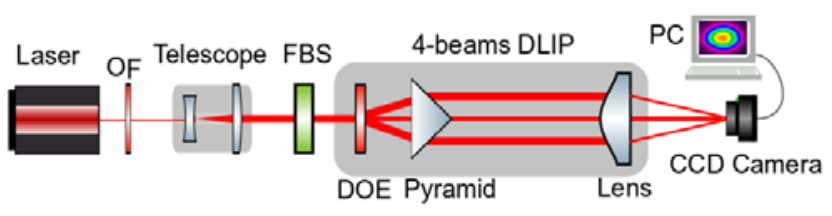

Fig. 3. Experimental setup for the investigation of the FBS element in combination with a DLIP optical configuration.

\section{Results and discussion}

The impact of the FBS element on the resulting DLIP pattern, the tolerance of the experimental setup to misalignments as well as the effect of optical aberrations on the beam wave front has been studied in a set of experiments.

First, the $0^{\text {th }}$ and the $1^{\text {st }}$ order top-hat beam profile including the interference pattern were obtained and the effect of beam collimation on the resulting beam profile was examined. Second, the periodicity of linear defects was investigated by introducing well-defined misalignments in the DLIP optical setup. The propagation of these defects from Gaussian beam profile to top-hat profile was simulated employing an optical ray-tracing approach and verified experimentally. 


\subsection{Interference top-hat intensity distribution}

The beam profile of the used ns-pulsed laser was measured by the CCD camera at the targeted position of the FBS element and adjusted to a beam diameter of $4.0 \mathrm{~mm}$ (at $1 / \mathrm{e}^{2}$ level) by optimizing the beam expander (see Fig. 4b). In the 4-beam DLIP setup, the distance between DOE and pyramid was set to $55 \mathrm{~mm}$ which results in an overlapping angle $\theta$ of $7.13^{\circ}$ and a spatial period $\Lambda$ of $6.0 \mu \mathrm{m}$.
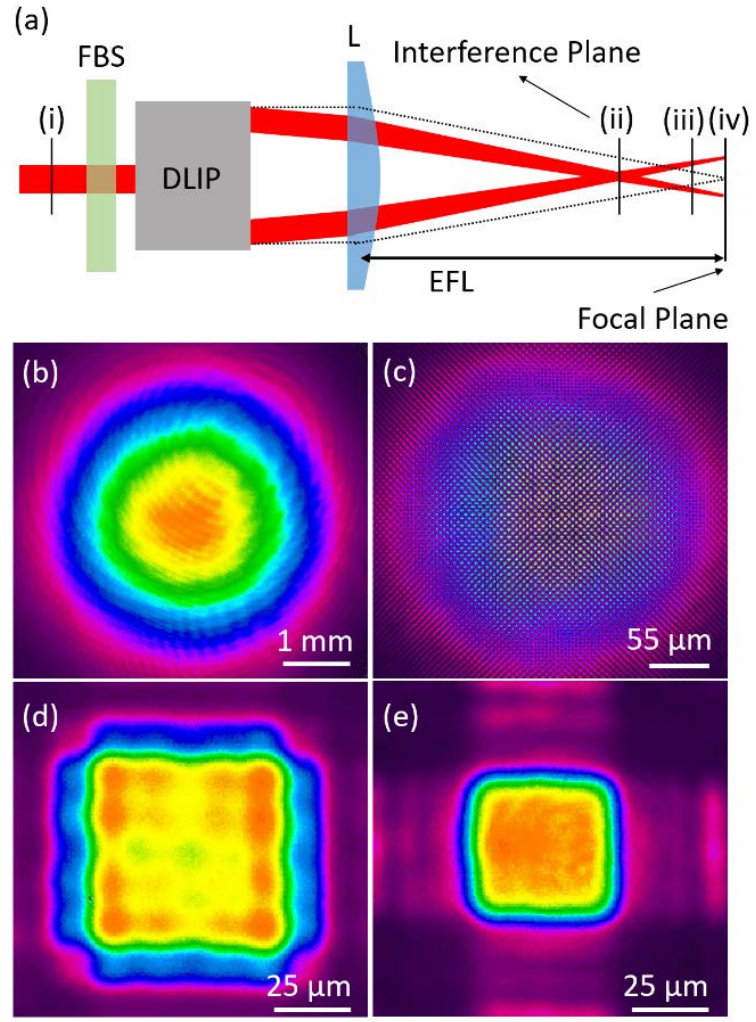

Fig. 4. Beam profiles measured by a CCD camera at different positions of the experimental setup (a); (b) Gaussian beam profile at input (i), (c) dot-like interference pattern at interference plane (ii), (d) $1^{\text {st }}$ order square-shaped flat top at position (iii), and (e) $0^{\text {th }}$ order square-shaped flat-top intensity distribution at position (iv).

In general, when the interference plane is near the focal plane, the resulting spot diameter is small with a characteristic size in the range of the diffraction limit. However, this is not preferred during DLIP processing since working in focus is typically accompanied by uncontrolled melting due to high laser fluences as well as low processing speeds due to the small spot sizes. Consequently, optimal processing requires a working position out of focus to achieve welldefined interference patterns as well as high throughputs.

This can be realized by achieving a slightly noncollinear orientation of the laser beams with respect to each other before the focusing lens (see Fig. 4a) which shifts the interference zone above the focusing plane. However, no top-hat intensity distribution was observed at the interference plane since the FBS element is designed to obtain the top-hat intensity distribution at the focal position. As shown in Fig. 4c, the characteristic 4-beam interference pattern was obtained, but the envelope of the intensity distributions follows still a Gaussian distribution.

A closer analysis of the top-hat beam profiles at the focal plane (denoted as $0^{\text {th }}$ order top-hat profile, see Fig. 4e) and before the focal plane (denoted as $1^{\text {st }}$ order top-hat profile, see Fig. 4d) revealed that the $1^{\text {st }}$ order top-hat profile is two times larger than the $0^{\text {th }}$ order top-hat profile. Although the $1^{\text {st }}$ order top-hat profile exhibit stronger deviations in the areal uniformity of the laser energy, it is still interesting for alignment as well as processing since a larger area permits to achieve higher throughputs.
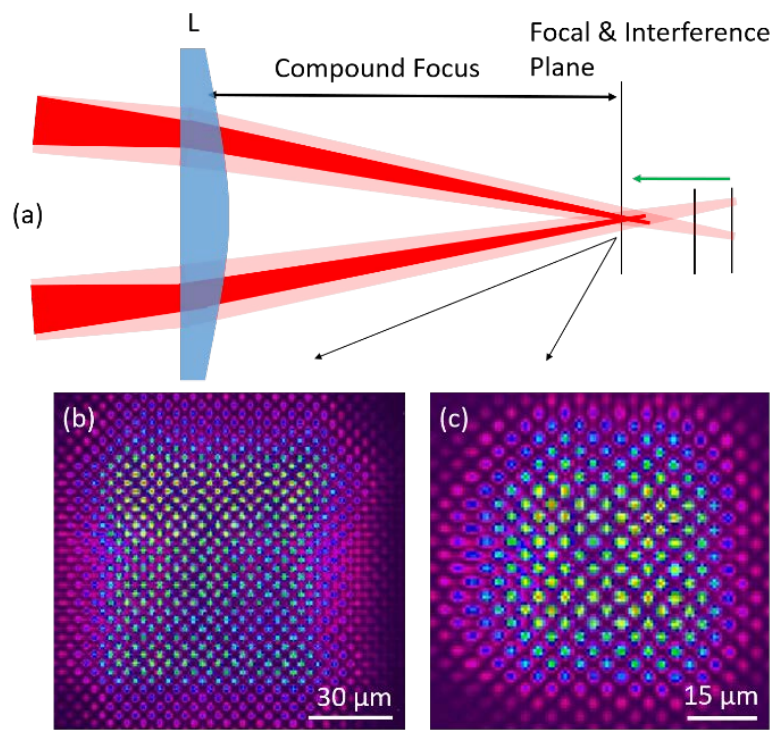

Fig. 5. Controlled de-collimation (a) of input laser beam allows to achieve (b) $1^{\text {st }}$ order top-hat profile at the interference plane and (c) $0^{\text {th }}$ order top-hat profile at the interference plane.

In order to control the position of the top-hat profile, which means to bring the top-hat intensity distribution into the interference plane, a controlled de-collimation of the input beam was performed by moving two lenses of the beam expander apart (see Fig. 5a). The resulting interference patterns are shown in Fig. $5 b$ and 5c, corresponding to the $0^{\text {th }}$ and $1^{\text {st }}$ order square-shaped top-hat intensity distribution, respectively. It turns out that although the FBS element is designed for a collimated input beam, its functionality is still preserved to a certain degree of de-collimation introduced by the telescope.

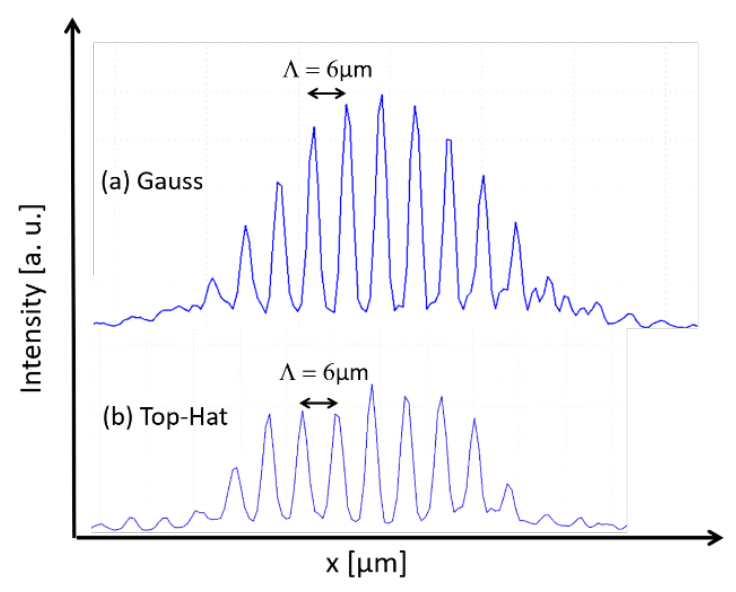

Fig. 6. CCD-measured intensity profiles at the interference plane introduced by a (a) Gaussian and (b) top-hat energy distribution.

The obtained interference patterns at the interference plane with and without the FBS element are exemplified in 
Fig. 6. For the Gaussian intensity distribution (without FBS), each measured interference maxima shows a different peak intensity. This can be changed by using the FBS element, which results in seven comparable interference maxima intensities (while two other maxima exhibit approximately half of the intensity).

\subsection{Alignment of the optical components}

In a second set of experiments, the impact of misalignment of the optical components on the resulting interference profile was investigated. Different misalignments were tested including the beam splitting element (DOE), the beam aligning element (Pyramid) as well as the focusing lens. Within all analyzed cases, it was found that misalignments in the pyramid tilt angle leads to the biggest effect on the measured interference intensity profiles. The influence of misalignments for the other elements will be investigated in more detail in the future.

$0^{\circ}$
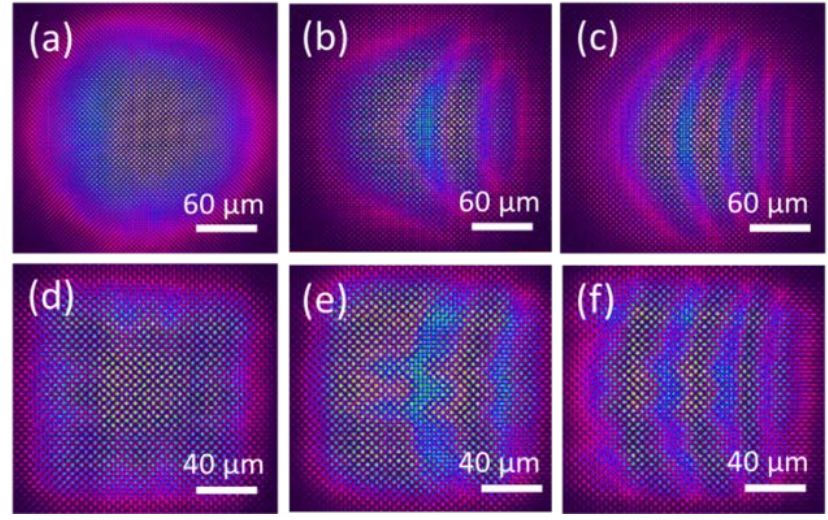

Fig. 7. Measured interference intensity profiles for different pyramid tilt angles (a-c) without and (d-f) with FBS element.

In Fig. $7 \mathrm{a}-\mathrm{c}$, the resulting interference profiles measured for different pyramid tilt angles are depicted for the DLIP setup without the FBS element. When the tilt angle of the pyramid equals $0^{\circ}$, the resulting interference intensity profile shows a uniform periodic appearance with Gaussian intensity envelope. However, when the pyramid is tilted by $0.28^{\circ}$ and $0.56^{\circ}$, the uniformity of the resulting profile is strongly disturbed (see Fig. 7b and Fig 7c) highlighting that a well-defined alignment of the optical components is crucial. Note that the uniformity of the interference intensity profile is modulated in the direction of the pyramid tilting while the intensity envelope of the spot preserves its Gaussian profile.

A similar outcome was also observed in the case of a top-hat beam distribution employing the FBS element. As depicted in Fig. 7d-f, small tilting angles of the pyramid results in strong irregularities of the interference intensity distributions. The observed distortion of the intensity distribution exhibits a distinct periodicity within the laser spot which depends on the pyramid tilting angle, as exemplified in Fig. 8.

To better understand the origin of the periodic intensity distortion, the influence of the pyramid tilting angle on the propagating sub-beams within the DLIP setup was analyzed in more detail. By using the optical design software Zemax (OpticStudio 15), the deflection of the sub-beams within the optical system is highlighted for a well-defined prism tilting angle (see Fig. 9). It can be seen that the tilting of the prism strongly affects the propagation of one of the four interfering beams (bottom beam in Fig. 9). As a result, the symmetry of the beam distribution after the focusing lens is disturbed.

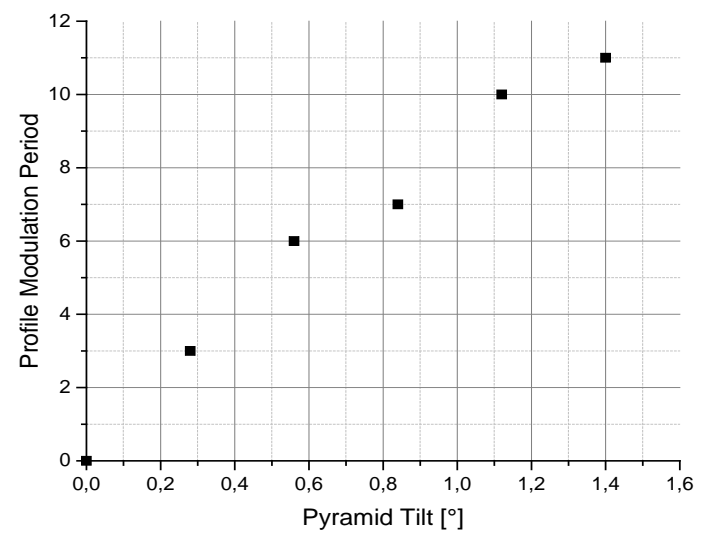

Fig. 8. Periodic modulation within the laser spot in dependence on the pyramid tilt angle.

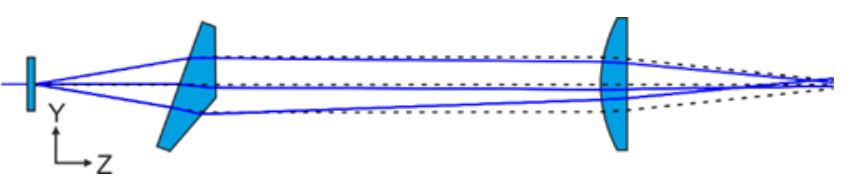

Fig. 9. Propagation of the beams when the pyramid is tilted by $0^{\circ}$ (dashed black line) and $20^{\circ}$ (solid blue line). The sketch was modeled using the software Zemax.

The impact of the asymmetrical beam distribution on the interference intensity distribution was supplemented by theoretical calculations based on Eq. (1). Therefore, 3 of the 4 beams were distributed symmetrically around the optical axis (with an intercepting angle of $\theta_{1-3}=6^{\circ}$ ) while the intercepting angle of the $4^{\text {th }}$ beam was increased by $1^{\circ}$ (which results in $\theta_{4}=7^{\circ}$ ). Both, the experimentally measured and theoretically calculated profiles are presented in Fig. 10.

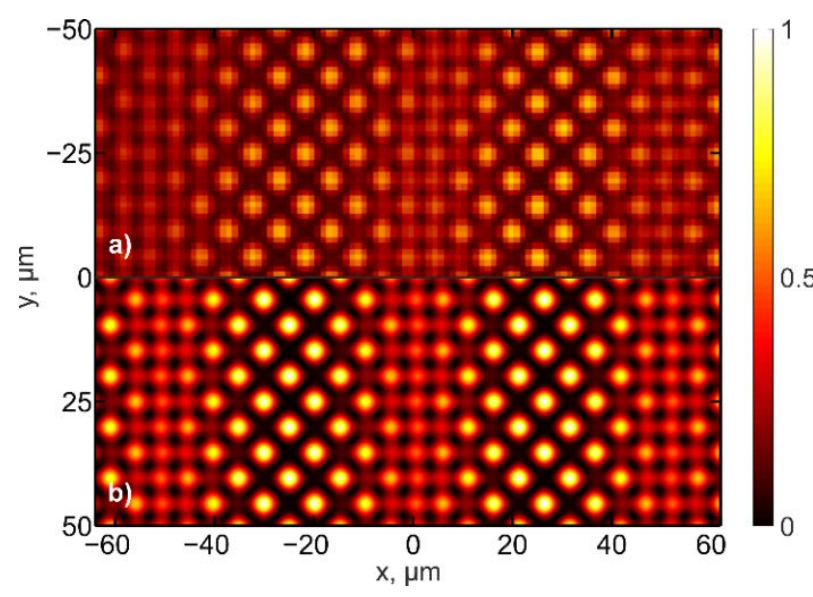

Fig. 10. (a) Experimentally measured and (b) theoretically calculated interference intensity profiles of a 4-beam DLIP with asymmetrical beam distribution around the optical axis $\left(\varphi_{1}=0^{\circ} ; \varphi_{2}=90^{\circ}\right.$; $\varphi_{3}=180^{\circ} ; \varphi_{4}=270^{\circ} ; \theta_{1}=\theta_{2}=\theta_{3}=6^{\circ} ; \theta_{4}=7^{\circ}$ ).

These two-interference intensity distribution show exceptional agreement with each other and show that asym- 
metry in the distribution of the interfering beams results in the modulation of the uniformity in the final beam profile.

\section{Summary}

The diffractive beam shaping optics, which applies FBS principle to generate square-shaped top-hat beam profile, was used with a 4-beam DLIP setup and analyzed with respect to compatibility. It was shown that in order to achieve interference profiles with square shaped top-hat energy distribution, the focal and interference plane has to be harmonized. This was done by a controlled disbalancing of the used beam expanding telescope system which results in a de-collimation of the input beam. It was also found that the 4-beam DLIP system is susceptible to optical misalignments leading to periodic modulations of the interference profile. The origin of the observed periodic modulations was traced back to tilts of the pyramid in the DLIP setup which highlights that a proper alignment is crucial for 4-beam interference. The insights were supplemented by theoretical simulations showing very good agreement with experimental measurements of the interference intensity profile. Finally, an optical system capable to produce square shaped 4-beam interference energy distributions with flat-top intensity envelope was proposed and demonstrated.

\section{Acknowledgments}

This project has received funding from the European Union's Horizon 2020 Framework Program for research and innovation under grant agreement no. 768701.

The work of A. F. Lasagni is supported by the German Research Foundation (DFG) under Excellence Initiative program by the German federal and state governments to promote top-level research at German universities.

\section{References}

[1] S. Nishimoto, B. Bhushan: RCS Adv., 3, (2013), 671.

[2] A. Kovalchenko, O. Ajayi, A. Erdemir, G. Fenske,I. Etsion: Tribol. Int., 38, (2005), 219.

[3] Y. Luo, X. Li, D. Zhang, Y. Liu: Surf. Eng., 32, (2016), 157.

[4] D.Y. Zhang, Y.H. Luo, X. Li, H.W. Chen: J. Hydrodyn., 23, (2011), 204

[5] A. Rosenkranz, L. Reinert, C. Gachot and F. Mücklich: Wear, 318, (2014), 49.

[6] V. Lang, T. Roch, A.F. Lasagni: Adv. Eng. Mater., 18, (2016), 1342.

[7] A. F. Lasagni, C. Gachot, K. E. Trinh, M. Hans, A. Rosenkranz, T. Roch, S. Eckhardt, T. Kunze. M. Bieda. D. Günther, V. Lang, F. Mücklich: Proc. SPIE, 10092, San-Francisco, (2017), pp. 1009211.

[8] C. E. Nebel, S. Christiansen, H. P. Strunk, B. Dahlheimer, U. Karrer, M. Stutzmann: Phys. Status Solidi A, 166, (1998), 667.

[9] F. Mücklich, A. Lasagni, C. Daniel: Intermetallics, 13, (2005), 437.

[10] K. Du: Proc. SPIE, 7202, San-Francisco, (2009), pp. 72020Q.

[11] A. I. Aguilar-Morales, S. Alamri, T. Kunze, A. F. Lasagni: Opt. Laser Technol., 107, (2018), 216.
[12] A. Lasagni and F. Mücklich: Appl. Surf. Sci., 247, (2005), 32.

[13] A. Lasagni and F. Mücklich: Applied Surf. Sci., 240, (2005), 214.

[14] A. Aktag, S. Michalski, L. Yue, R. D. Kirby, S. H. Liou: J. Appl. Phys., 99, (2006), 093901.

[15] L. Guo, H.-B. Jiang, R.-Q. Shao, Y.-L. Zhang, S.-Y. Xie: Carbon, 50, (2012), 1667.

[16] T. Kondo, S. Matsuo, S. Juodkazis, H. Misawa: Appl. Phys. Lett., 79, (2001), 725

[17] Y. Nakata: Adv. Opt. Technol., 5, (2016), 29.

[18] A. Rodriguez, M. Echeverría, M. Ellman, N. Perez, Y. K. Verevkin: Microelectron. Eng., 86, (2009), 937

[19] S. Indrišiūnasa, B. Voisiat, M. Gedvilas, G. Račiukaitis: Laser Applications, 29, (2017), 011501.

[20] C. Zwahr, B. Voisiat, A. Welle, D. Günther, A. F. Lasagni: Adv. Eng. Mater., 20, (2018), 1800160.

[21] E. Hecht, (Addison-Wesley, 2002), p.387.

[22] G. Račiukaitis, E. Stankevičius, P. Gečys, M. Gedvilas, C. Bischoff, E. Jäger, U. Umhofer, F. Völklein: J. Laser Micro / Nanoengineering, 6, (2011), 37.

[23] C. Bischoff, U. Rädel, U. Umhofer, E. Jäger: Proc. SPIE, 8600, San-Francisco, (2013) pp. 860024.

[24] S. Rung, C. Bischoff, E. Jäger, U. Umhofer, R. Hellmann: Proc. SPIE, 8967, San-Francisco, (2014) pp. 89670P.

[25] C. Bischoff, E. Jäger, U. Umhofer: Laser Technik J., 12, (2015), 53.

[26] A.F. Lasagni, U. S. Patent 9,764,424 B2 (2014)

(Received: June 25, 2018, Accepted: November 11, 2018) 